Title:

\title{
2 Drosophila Male Accessory Gland Displays Developmental Ground Plan of a Primitive Prostate
}

3

4

Running title:

8

9

10

11

12

13

14

\author{
Authors \\ Jaya Kumari ${ }^{1}$ and Pradip Sinha, ${ }^{1, *}$ \\ ${ }^{1}$ Department of Biological Sciences and Bioengineering \\ Indian Institute of Technology Kanpur \\ Kanpur 208016, India \\ *Author for correspondence: pradips@iitk.ac.in
}


Developmental ground plan of Drosophila male accessory gland

15 Drosophila Male Accessory Gland Displays Developmental Ground Plan of a Primitive Prostate

16 Abstract

17 Conservation of developmental genetic toolkits of functionally comparable organs from disparate

18 phyla reveals their deep homology, which may help overcome the challenges of their confounding categorization as either homologous or analogous organs. A male accessory sexual organ in mammals, prostate, for instance, is anatomically disparate from its phylogenetically distant counterpart—-the male accessory gland (MAG)—in insects like Drosophila. By examining a select set of toolkit gene expression patterns, here we show that Drosophila MAG displays deep homology with the mammalian prostate. Like mammalian prostate, MAG morphogenesis is marked by recruitment of fibroblast growth factor receptor, FGFR, a tubulogenesis toolkit signaling pathway, starting early during its adepithelial genesis. Specialization of the individual domains of the developing MAG tube on the other hand is marked by expression of a posterior Hox gene transcription factor, Abd-B, while Hh-Dpp signaling marks its growth. Drosophila MAG thus reveals developmental design of unitary bud-derived tube-a ground plan that appears to have been reiteratively co-opted during evolutionary diversification of male accessory sexual organs across distant phylogeny.

\section{Introduction}

Conservation of genetic toolkits—-the molecular architects of animal body plan—has helped reveal underlying developmental principles across wide phylogenetic spectrum (Carroll et al., 2001; Shubin et al., 2009). Recruitment of Pax6 transcription factor for photosensory organs in all bilateria from annelid worms, invertebrates to highly evolved vertebrate eyes being one of the oft-cited and most elegant illustrations of conservation of developmental toolkit genes (Gehring and Seimiya, 2010; Halder et al., 1995; Schwab, 2018). Conservation of developmental toolkit genes—also termed as deep homology (Shubin et al., 1997; Shubin et al., 2009)—has further helped decipher evolutionary relatedness based on genetic blueprints. Deep homology, when revealed, permits deconstructions of complex anatomical features of an organ into its independent or modular components and trace their evolutionary changes (Brandon, 2005; Rasskin-Gutman, 2005). Modularity, rather its breakaways therefore underlie evolutionary diversification from a primitive developmental ground plan of an organ.

The mammalian prostate is derived from a common endodermal embryonic primordium of reproductive and urinary organs termed as the urogenital sinus (UGS) (Marker et al., 2003; Shapiro et al., 2004). Specification of prostatic UGS at its pre-bud stage is followed by its budding, bud elongation, branching and, finally, canalization within each of its primary and secondary branches that create their individual lumens (for review, see Cunha et al., 2018). Epithelial linings of these lumens differentiate into two secretory cell types: luminal and basal (for review, see Marker et al., 2003). Cell 
Developmental ground plan of Drosophila male accessory gland

49 fate specification of the prostatic primordium is marked by gain of homeodomain transcription

50 factors, such as Nkx3.1 (for review, see Prins and Putz, 2008), while recruitment of fibroblast growth

51 factor (FGF) and sonic hedgehog (Shh) signalings regulate their growth and branching (Doles et al.,

52 2006; Donjacour et al., 2003; Le et al., 2020; Lin et al., 2007). Posterior Hox genes: for instance,

53 Hoxb13 induces cell differentiation in the luminal cell (Economides and Capecchi, 2003; Huang et al.,

54 2007).

55 Prostate-like protein-rich seminal fluid secreting organs termed male accessory glands (MAG) are 56 also present in invertebrates, as in the dipteran insect, the fruit fly, Drosophila (Findlay et al., 2008;

57 Gilany et al., 2015; Ravi Ram and Wolfner, 2007; Verze et al., 2016). By contrast to endodermal 58 mammalian prostate, Drosophila MAG is mesodermal in origin and represents a paired tubular organ

59 (Ahmad and Baker, 2002). It originates from a common adepithelial primordium - which develops in 60 close apposition with the ectodermal male genital disc epithelium — and gives rise to both MAG and

61 seminal vesicle (SV). Drosophila counterpart of mammalian FGFR, namely, Breathless (Btl) marks

62 the common embryonic MAG-SV primordium (Ahmad and Baker, 2002). Adult MAG is a relatively

63 simple tubular organ, its lumen is formed by squamous epithelium marked by two differentiated cell

64 types: main and secondary, and is overlaid by circular rings of contractile muscles (Bairati, 1968;

65 Susic-Jung et al., 2012).

66 Mammalian prostate is proposed to have appeared nearly 65 million years ago (Coffey, 2001) and its

67 evolutionary origin appears to be independent of that of its Drosophila counterpart, MAG. Here we

68 show that MAG development in Drosophila reveals an essential unitary prostatic tubule and is formed

69 by shared genetic toolkits, which are also recruited during mammalian prostate development.

70 Drosophila MAG therefore reveals a primitive and modular developmental ground plan, which

71 appears to have been co-opted across distant phyla.

72 Results and Discussion

\section{Spatial coordinates of developing MAG-SV and genital primordia}

74 In third instar male larvae, a group of mesodermal adepithelial cells-marked by expression of

75 Breathless (Btl)/FGF receptor (FGFR) -migrate and lodge onto the epithelium of the genital

76 imaginal disc and serve as a common primordium of seminal vesicle (SV) and male accessory gland

77 (MAG) (Ahmad and Baker, 2002) (see Figure 1A). The MAG-SV primordium thus develops in close

78 apposition with the genital disc from third larval instar till puparium formation (Figure 1A-C). Optical

79 cross-section further revealed that MAG-SV primordium is surrounded by the genital disc epithelium

80 (Figure 1A'-C', zoomed cross-section in B'"), the latter marked by a Drosophila posterior Hox gene

81 Abd-B (Figure1C', zoomed cross-section in C') (Estrada and Sánchez-Herrero, 2001; de Navas et al.,

82 2006) and express its genetic toolkits like engrailed (en) and cubitus interruptus (ci) (Figure 1D),

83 which mark its presumptive anterior-posterior compartments wherein patched (ptc) (Figure 1E) 
Developmental ground plan of Drosophila male accessory gland

$84 \quad$ straddles their boundary while signaling pathways like Wingless $(\mathrm{Wg})$ (Figure $1 \mathrm{~F}$ ) and

85 Decapentapelagic (Dpp) (Figure 1G) regulate its morphogenesis (Casares et al., 1997; Chen and

86 Baker, 1997; Freeland and Kuhn, 1996). The mesodermal MAG-SV and the ectodermal genital

87 primordia thus develop in close apposition prefiguring their mutual contacts in the adult.

Mammalian (rodent) prostate develops from multiple buds that spatially form its bilaterally symmetrical anterior, dorso-lateral, and ventral lobes (Lin et al., 2003; Marker et al., 2003; Timms, morphogenesis; its expression being robust at the distal tips of these branching buds revealing its critical role as a toolkit signaling pathway during rodent prostate development (Huang et al., 2005), Thus, upon conditional knockdown of FGFR, the anterior and the ventral lobes are selectively lost while ductal patterning of dorso-lateral lobe compromised (Lin et al., 2007). In all prostatic lobes, the posterior Hox, Hoxb13 is specifically expressed in the ductal epithelium, its highest expression being the luminal cells (Economides and Capecchi, 2003; Huang et al., 2007). MAG-SV primordium at late $3^{\text {rd }}$ instar, too, was shown to express the Bt1/FGFR (Ahmad and Baker, 2002) toolkit signaling pathway while the sole Drosophila posterior Hox gene, Abd-B (Coiffier et al., 2008) is selectively expressed in the differentiated secondary cells of the adult MAG (Gligorov et al., 2013) (48h after eclosion (AE), Figure 2A).

These parallels between mammalian prostate and Drosophila MAG therefore hinted at similarities in their developmental ground plan. To further explore and elaborate this ground plan of the developing MAG, we examined Btl (btl-Gal4>UAS-GFP) and Abd-B during the entire course of pupal morphogenesis of MAG. We noticed that Bt1/FGFR robustly marks the MAG bud early during its morphogenesis (30-50h APF, Figure 2B-D). Further, around 50h APF, Bt1/FGFR displays robust expression in a group of distal cells (Figure 2D, see zoomed distal tip 50h APF) and, extinguishes, shortly ahead of the conclusion of pupal development (Figure 2E-H, 60-90h APF) suggesting its role during MAG bud growth and morphogenesis rather than in the adult gland. bud (30-50h APF, Figure 2B-D), unlike its expression in the developing SV throughout its budding and growth phase (Figure $2 \mathrm{~B}-\mathrm{H}$ ). Abd-B first appeared in the presumptive secondary cells of the MAG at 50h AFP (Figure 2D, see zoomed distal tip at 50h APF). Interestingly, Abd-B also initially expresses in the MAG main cells at 60h APF (Figure 2D, see zoomed distal tip at 60h APF) but tips); thus its dynamic pattern of expression is marked by two characteristics: that is, its transient but cells. 
Developmental ground plan of Drosophila male accessory gland

119 Taken together, Bt1/FGFR appears to be a primary toolkit for MAG-SV bud growth —a possibility

120 that was further strengthened by the observation that knockdown ( $c i-G a l 4>b t l-R N A i)$ of Bt1/FGFR truncated growth of the MAG (Figure 2J-K) with accompanying loss of its cell differentiation as revealed, for instance, by loss of development of the Abd-B-expressing secondary cells (Figure 2L). These features are reminiscent of fallouts of FGFR knockdown in prostate precursor cells in embryonic mouse, which completely abolishes growth of its anterior and ventral lobes and compromises growth of its dorso-lateral prostatic lobes as well as their cell fate acquisitions via. Hoxd13 expression or other markers such as Bmp4 and TGF- $\beta$ (Lin et al., 2007).

Bt1/FGFR signaling and posterior Hox toolkit expression pattern in the highly branched and multilobular mammalian prostate (Huang et al., 2007) therefore appears to be a reiterative deployment of a simple tubular developmental ground plan of Drosophila MAG revealing deep homology of such accessory sexual organs from distant phylogeny.

\section{Hedgehog-Dpp signaling axis in MAG morphogenesis}

In mouse prostate development, Shh signaling activity appears at budding, marks the nascent prostatic bud, declines as growth proceeds, and is seen in the gland of the adult gland at very low levels (Doles et al., 2006; Lamm et al., 2002). The ligand (Shh) is enriched at the distal prostatic tips of the epithelial bud, the receptor (Ptc), transcription factor (Gli) and downstream targets (e.g. Bmp4) are prominently expressed in the surrounding UGS mesenchyme (Lamm et al., 2001; Lamm et al., 2002; Podlasek et al., 1999; Pu et al., 2004). Developmental design reflected by Shh signaling with respect to prostate development, however, remains ambiguous owing in part to genetic redundancy: for instance, Gli1 and Gli3 may compensate for Gli2 loss in UGS tissues (Doles et al., 2006). It has, however, not been ascertained yet if MAG displays expression of the Hh/Shh signaling tool kit, reminiscent of that seen in prostate.

We thus examined the expression of Hh pathway members in the MAG bud; $\mathrm{Ci}$ ( $c i$-Gal4>UAS-GFP), Ptc (ptc-Gal4>UAS-GFP) and Dpp (dpp-Gal4>UAS-GFP)—a Hh target, and mammalian Bmp counterpart—during the course of its pupal morphogenesis. While $c i>G F P$ expression may not represent its actual activation - which is denoted by its cleaved form Ci-75 (Huangfu and Anderson, 2006; Price and Kalderon, 1999) — we consider it relevant for our purpose. We noted Ci expression around 20h APF: that is, during budding of MAG, (Figure 3A, see zoomed box, A'), which continued through its bud growth (Figure 3D, 30h APF) and cell differentiation stages (Figure 3G, 50h APF) and was finally extinguished in the MAG (Figure 3J, 90h APF). The spatio-temporal expressions of Hh receptor Ptc, too, marked the budding (20h APF, Figure 3B zoomed box), bud growth (30h APF, Figure $3 \mathrm{E}$ ) and cell differentiation stages (50h APF, Figure $3 \mathrm{H}$ ) albeit more prominently at the distal end compared to that of $\mathrm{Ci}$. Moreover, unlike $\mathrm{Ci}$ that extinguishes by the end of pupal development, Ptc was retained in the secondary cells (90h APF, Figure 3K) suggesting its further roles in these cells 
Developmental ground plan of Drosophila male accessory gland

154 in the adult gland. Hh target, Dpp, however, did not mark the nascent bud (20h APF, zoomed bud 155 Figure 3C). It faintly appeared at the distal end during bud growth (30h APF, Figure 3F) and cell 156 differentiation (50h APF, Figure 3I). Its robust expression was noted in the secondary cells by the 157 completion of pupal MAG morphogenesis (90h APF, Figure 3L).

158 These expression patterns during the course of MAG development therefore suggest Hh signaling 159 requirement during bud growth stage and cell fate specification although Bt1/FGFR may be the 160 earliest crucial toolkit. While these inferences need critical functional evaluation, what appears most relevant to the current investigation is the fact that $\mathrm{Hh}$ signaling pattern in MAG development does appear reminiscent of that of prostate on the following general account: namely, its appearance in the nascent bud, followed by distal enrichment as the tube grows and, finally, decline in its expression upon formation of the tube.

\section{Conclusion}

Co-options of common development ground plans via recruitment of shared toolkit genes mirror deep homology amongst organs of diverse phyla (Shubin et al., 1997; Shubin et al., 2009). Our study suggested that the developmental ground plan of MAG as a simplified tube also recurs during mammalian prostatic bud development, which are branched and turn into tubules via. canalization (Figure 4). These parallels reveal that in a blind watch-maker's (Dawkins, 1986) analogy of evolution, common toolkits are co-opted across distant phylogenies, presumably de novo, which recreate a ground plan for development of organs. Reiterative deployment of this ground plan further results in modular designs of these organs, while deviations in these modules adds the diversities encountered across distantly related phylogenies (Wagner, 2007; Wagner and Altenberg, 1996). Binucleation and cuboidal-to-squamous transitions in the MAG epithelium (Taniguchi et al., 2014; Wilson et al., 2017) and columnar luminal cells, besides appearance of progenitor neuroendocrine cells in the mammalian prostate (Marker et al., 2003; Toivanen and Shen, 2017) represent individual phylogenetic peculiarities of these accessory sexual organs. Our study further suggests that-these evolutionary lineage specific peculiarities notwithstanding-MAG could also serve a model of a primitive prostate and therefore it could indeed be examined for modelling prostatic diseases, including cancers, for instance (Rambur et al., 2020).

\section{Material and Methods}

Collection and staging of pupal samples. Male pupae were identified by their prominent ovoid gonads. Males of the required genotype were selected by examining GFP expression under a fluorescent microscope. The 0h was decided as previously described (Bainbridge and Bownes, 1981). The maximum time variation may range \pm 30 minutes amongst individuals of a given stage. Collected Oh samples were allowed to grow at $25 \pm 1 \square$ in a petri-dish with moist filter paper until the time of dissection. At least 5 individuals of a genotype were dissected for a given time point. 
Developmental ground plan of Drosophila male accessory gland

189 Dissection of pupal MAG samples. Pupal dissection for 0-20h APF samples were done by excising the lower $1 / 3^{\text {rd }}$ of the pupae using dissection scissors in cold PBS. The genital disc was gently pushed out of the pupal cover using insulin syringes. For 30-70h pupal samples, first a cut was made at the abdomen-thorax junction. The reproductive structures within the abdomen were gently pushed out using insulin syringes. The tissue was cleared of abdominal fat and lipids. The pharate stage animals between 80-90h APF were taken out of the pupal case. MAG was isolated by gently removing the external genitalia using a pair of insulin syringes similar to adult dissection.

Immunofluorescence staining and microscopy. Standard immunofluorescence protocol was

followed. In brief, samples were fixed in 4\% paraformaldehyde for 35 minutes, washed with PBS and incubated with the desired primary antibody overnight. The samples were washed, blocked in bovine serum albumin for an hour, before adding secondary antibody. Samples were counterstained with TOPRO-3 (Invitrogen) and/or Phalloidin. Primary antibodies used: Mouse anti-En (1:50), Mouse antiAbd-B (1:50), Mouse anti-FasIII (1:100); Phalloidin 555, 633 (1:100), TOPRO (1:500). Finally, the prepared samples were mounted in Vectshield or Prolong Gold for imaging. Images were acquired using a Leica confocal SP5 system and processed using LAS AF software.

Key Resources Table:

\begin{tabular}{|l|l|l|}
\hline REAGENT/RESOURCE & SOURCE & IDENTIFIER \\
\hline & & \\
\hline Anti-Engrailed/Invected & DSHB* & $4 \mathrm{D} 9$ \\
\hline Anti-Abd-B & DSHB & 1 A2E9 \\
\hline Anti-FasIII & DSHB & $7 \mathrm{G} 10$ \\
\hline Alexa Fluor Phalloidin555 & Invitrogen & A34055 \\
\hline Alexa Fluor Phalloidin 633 & Invitrogen & A22284 \\
\hline TO-PRO-3 & Invitrogen & S33025 \\
\hline *Developmental Studies Hybridoma Bank, University of Iowa & \\
\hline Experimental models: Transgenic fly lines & & \\
\hline Abd-B-Gal4 & Gift from Dr.Rakesh Mishra & ---------- \\
\hline$b t l-G a l 4$ & BDSC* & BDSC:78328 \\
\hline ci-Gal4 & Gift from Dr.Ishwar Hariharan & --------- \\
\hline$d p p$-Gal4 & BDSC & \\
\hline ptc-Gal4 & BDSC & BDSC:2017 \\
\hline wg-Gal4 & Gift from Dr. Jean-Paul Vincent & --------- \\
\hline
\end{tabular}


bioRxiv preprint doi: https://doi.org/10.1101/2021.03.20.436254; this version posted March 20, 2021 . The copyright holder for this preprint (which was not certified by peer review) is the author/funder. All rights reserved. No reuse allowed without permission.

Developmental ground plan of Drosophila male accessory gland

\begin{tabular}{|l|l|l|}
\hline$U A S-b t l-R N A i$ & BDSC & BDSC: 40871 \\
\hline$U A S-G F P$ & BDSC & BDSC:4776 \\
\hline *Bloomington Drosophila Stock Centre, Indiana University & \\
\hline \multicolumn{2}{|l|}{} \\
\hline Software & & \\
\hline Leica Application Suite, AF, 2.6.0 build & Leica & \\
\hline
\end{tabular}

206

Funding:

This investigation was supported by a research grant from DST to Pradip Sinha (Project no.

EMR/2016/006723).

\section{Conflict of interest:}

There authors declare no conflict of interest.

\section{References:}

Ahmad, S. M. and Baker, B. S. (2002). Sex-specific deployment of FGF signaling in Drosophila recruits mesodermal cells into the male genital imaginal disc. Cell 109, 651-661.

Bairati, A. (1968). Structure and Ultrastructure of the Male Reproductive System in Drosophila Melanogaster Meig. Monit. Zool. Ital. - Ital. J. Zool. 2, 105-182.

Brandon (2005). Modularity: Understanding the Development and Evolution of Natural Complex Systems. In: Evolutionary Modules: Conceptual Analyses and Empirical Hypotheses, 51-60. Cambridge: The MIT Press.

Carroll, S. B., Grenier, J. K. and Weatherbee, S. D. (2001). From DNA to Diversity: Molecular Genetics and the Evolution of Animal Design. In: The Genetic Toolkit for Development, 1753. Blackwell Science Ltd, Oxford.

Casares, F., Sánchez, L., Guerrero, I. and Sánchez-Herrero, E. (1997). The genital disc of Drosophila melanogaster. $\square$ : I. Segmental and compartmental organization. Dev. Genes Evol. 207, 216-228.

Chen, E. H. and Baker, B. S. (1997). Compartmental organization of the Drosophila genital imaginal discs. Development 124, 205-218.

Coffey, D. S. (2001). Similarities of prostate and breast cancer: Evolution, diet, and estrogens. Urology 57, 31-38.

Coiffier, D., Charroux, B. and Kerridge, S. (2008). Common functions of central and posterior Hox genes for the repression of head in the trunk of Drosophila. Development 135, 291-300. 
Cunha, G. R., Vezina, C. M., Isaacson, D., Ricke, W. A., Timms, B. G., Cao, M., Franco, O. and Baskin, L. S. (2018). Development of the human prostate. Differ. Res. Biol. Divers. 103, 24 45.

Dawkins, R. (1986). The Blind Watchmaker. In: Explaining the Very Improbable, 1-18. WW Norton \& Company. New York.

de Navas, L., Foronda, D., Suzanne, M. and Sánchez-Herrero, E. (2006). A simple and efficient method to identify replacements of P-lacZ by P-Gal4 lines allows obtaining Gal4 insertions in the bithorax complex of Drosophila. Mech. Dev. 123, 860-867.

Doles, J., Cook, C., Shi, X., Valosky, J., Lipinski, R. and Bushman, W. (2006). Functional compensation in Hedgehog signaling during mouse prostate development. Dev. Biol. 295, 1325.

Donjacour, A. A., Thomson, A. A. and Cunha, G. R. (2003). FGF-10 plays an essential role in the growth of the fetal prostate. Dev. Biol. 261, 39-54.

Economides, K. D. and Capecchi, M. R. (2003). Hoxb13 is required for normal differentiation and secretory function of the ventral prostate. Development 130, 2061-2069.

Findlay, G. D., Yi, X., MacCoss, M. J. and Swanson, W. J. (2008). Proteomics Reveals Novel Drosophila Seminal Fluid Proteins Transferred at Mating. PLoS Biol. 6, e178.

Freeland, D. E. and Kuhn, D. T. (1996). Expression patterns of developmental genes reveal segment and parasegment organization of D. melanogaster genital discs. Mech. Dev. 56, 61-72.

Gehring, W. and Seimiya, M. (2010). Eye evolution and the origin of Darwin's eye prototype. Ital. J. Zool. 77, 124-136.

Gilany, K., Minai-Tehrani, A., Savadi-Shiraz, E., Rezadoost, H. and Lakpour, N. (2015). Exploring the Human Seminal Plasma Proteome: An Unexplored Gold Mine of Biomarker for Male Infertility and Male Reproduction Disorder. J. Reprod. Infertil. 16, 61-71.

Gligorov, D., Sitnik, J. L., Maeda, R. K., Wolfner, M. F. and Karch, F. (2013). A Novel Function for the Hox Gene Abd-B in the Male Accessory Gland Regulates the Long-Term Female Post-Mating Response in Drosophila. PLOS Genet. 9, e1003395.

Halder, G., Callaerts, P. and Gehring, W. J. (1995). Induction of ectopic eyes by targeted expression of the eyeless gene in Drosophila. Science 267, 1788-1792.

Huang, L., Pu, Y., Alam, S., Birch, L. and Prins, G. S. (2005). The role of Fgf10 signaling in branching morphogenesis and gene expression of the rat prostate gland: lobe-specific suppression by neonatal estrogens. Dev. Biol. 278, 396-414.

Huang, L., Pu, Y., Hepps, D., Danielpour, D. and Prins, G. S. (2007). Posterior Hox Gene Expression and Differential Androgen Regulation in the Developing and Adult Rat Prostate Lobes. Endocrinology 148, 1235-1245.

Huangfu, D. and Anderson, K. V. (2006). Signaling from Smo to Ci/Gli: conservation and divergence of Hedgehog pathways from Drosophila to vertebrates. Development 133, 3-14.

Lamm, M. L., Podlasek, C. A., Barnett, D. H., Lee, J., Clemens, J. Q., Hebner, C. M. and Bushman, W. (2001). Mesenchymal factor bone morphogenetic protein 4 restricts ductal budding and branching morphogenesis in the developing prostate. Dev. Biol. 232, 301-314. 


\author{
Lamm, M. L., Catbagan, W. S., Laciak, R. J., Barnett, D. H., Hebner, C. M., Gaffield, W., \\ Walterhouse, D., Iannaccone, P. and Bushman, W. (2002). Sonic hedgehog activates \\ mesenchymal Gli1 expression during prostate ductal bud formation. Dev. Biol. 249, 349-366.
}

Le, V., He, Y., Aldahl, J., Hooker, E., Yu, E.-J., Olson, A., Kim, W. K., Lee, D.-H., Wong, M., Sheng, R., et al. (2020). Loss of androgen signaling in mesenchymal sonic hedgehog responsive cells diminishes prostate development, growth, and regeneration. PLOS Genet. 16, e1008588.

Lin, T.-M., Rasmussen, N. T., Moore, R. W., Albrecht, R. M. and Peterson, R. E. (2003). Regionspecific inhibition of prostatic epithelial bud formation in the urogenital sinus of C57BL/6 mice exposed in utero to 2,3,7,8-tetrachlorodibenzo-p-dioxin. Toxicol. Sci. Off. J. Soc. Toxicol. 76, 171-181.

Lin, Y., Liu, G., Zhang, Y., Hu, Y.-P., Yu, K., Lin, C., McKeehan, K., Xuan, J. W., Ornitz, D. M., Shen, M. M., et al. (2007). Fibroblast growth factor receptor 2 tyrosine kinase is required for prostatic morphogenesis and the acquisition of strict androgen dependency for adult tissue homeostasis. Development 134, 723-734.

Marker, P. C., Donjacour, A. A., Dahiya, R. and Cunha, G. R. (2003). Hormonal, cellular, and molecular control of prostatic development. Dev. Biol. 253, 165-174.

Podlasek, C. A., Barnett, D. H., Clemens, J. Q., Bak, P. M. and Bushman, W. (1999). Prostate development requires Sonic hedgehog expressed by the urogenital sinus epithelium. Dev. Biol. 209, 28-39.

Price, M. A. and Kalderon, D. (1999). Proteolysis of cubitus interruptus in Drosophila requires phosphorylation by protein kinase A. Development. 126, 4331-4339.

Prins, G. S. and Putz, O. (2008). Molecular signaling pathways that regulate prostate gland development. Differ. Res. Biol. Divers.76, 641-659.

Pu, Y., Huang, L. and Prins, G. S. (2004). Sonic hedgehog-patched Gli signaling in the developing rat prostate gland: lobe-specific suppression by neonatal estrogens reduces ductal growth and branching. Dev. Biol. 273, 257-275.

Rambur, A., Lours-Calet, C., Beaudoin, C., Buñay, J., Vialat, M., Mirouse, V., Trousson, A., Renaud, Y., Lobaccaro, J.-M. A., Baron, S., et al. (2020). Sequential Ras/MAPK and $\mathrm{PI} 3 \mathrm{~K} / \mathrm{AKT} / \mathrm{mTOR}$ pathways recruitment drives basal extrusion in the prostate-like gland of Drosophila. Nat. Commun. 11, 2300.

Rasskin-Gutman, D.(2005).Modularity: Understanding the Development and Evolution of Natural Complex Systems. In: Modularity: Jumping forms within morphospace, 207-219. Cambridge, MA: MIT Press.

Ravi Ram, K. and Wolfner, M. F. (2007). Seminal influences: Drosophila Acps and the molecular interplay between males and females during reproduction. Integr. Comp. Biol. 47, 427-445.

Schwab, I. R. (2018). The evolution of eyes: major steps. The Keeler lecture 2017: centenary of Keeler Ltd. Eye 32, 302-313.

Shapiro, E., Huang, H., McFADDEN, D. E., Masch, R. J., Ng, E., Lepor, H. and Wu, X.-R. (2004). The Prostatic Utricle is not a Mullerian Duct Remnant: Immunohistochemical Evidence for a Distinct Urogenital Sinus Origin. J. Urol. 172, 1753-1756. 
Shubin, N., Tabin, C. and Carroll, S. (1997). Fossils, genes and the evolution of animal limbs. Nature 388, 639-648.

Shubin, N., Tabin, C. and Carroll, S. (2009). Deep homology and the origins of evolutionary novelty. Nature 457, 818-823.

Susic-Jung, L., Hornbruch-Freitag, C., Kuckwa, J., Rexer, K.-H., Lammel, U. and RenkawitzPohl, R. (2012). Multinucleated smooth muscles and mononucleated as well as multinucleated striated muscles develop during establishment of the male reproductive organs of Drosophila melanogaster. Dev. Biol. 370, 86-97.

Taniguchi, K., Kokuryo, A., Imano, T., Minami, R., Nakagoshi, H. and Adachi-Yamada, T. (2014). Isoform-specific functions of Mud/NuMA mediate binucleation of Drosophilamale accessory gland cells. BMC Dev. Biol. 14, 46.

Timms, B. G. (2008). Prostate development: a historical perspective. Differ. Res. Biol. Divers. 76, 565-577.

Toivanen, R. and Shen, M. M. (2017). Prostate organogenesis: tissue induction, hormonal regulation and cell type specification. Development. 144, 1382-1398.

Verze, P., Cai, T. and Lorenzetti, S. (2016). The role of the prostate in male fertility, health and disease. Nat. Rev. Urol. 13, 379-386.

Wagner, G. P. (2007). The developmental genetics of homology. Nat. Rev. Genet. 8, 473-479.

Wagner, G. P. and Altenberg, L. (1996). Perspective: Complex Adaptations and the Evolution of Evolvability. Evol. Int. J. Org. Evol. 50, 967-976.

Wilson, C., Leiblich, A., Goberdhan, D. C. I. and Hamdy, F. (2017). The Drosophila Accessory Gland as a Model for Prostate Cancer and Other Pathologies. Curr. Top. Dev. Biol. 121, 339375.

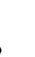

9


Developmental ground plan of Drosophila male accessory gland

\section{Figure Legends}

\section{Figure 1. MAG-SV and genital primordia develop in close apposition}

(A-C) GFP marked MAG-SV primordium in the backdrop of genital imaginal disc at (A) $3^{\text {rd }}$ instar and (B-C) 0h APF. Zoomed box A' (Actin, grey) depicts remodelling of the imaginal disc at the $3^{\text {rd }}$ instar, while $b t l-G a l 4>U A S-G F P$ marked MAG-SV primordial cells lodge. Zoomed boxes (B') show the clustered MAG-SV primordium, (B') show the apposition of genital and MAG-SV primordia in $\mathrm{X}-\mathrm{Z}$ section, and (C', C') the characteristic large nuclei of MAG-SV primordial cells. X-Z view at $0 \mathrm{~h}$ APF in B' and C' is further enlarged below.(D-G) Genital disc pattering genes displayed by their psuedo-colored GFP and immunostaining (En) depict the spatial position of overlaying MAG-SV primordium, which is denoted by broken lines. Scale bar: $100 \mu \mathrm{m}$ Abbreviations: AEL=After egg laying, $\mathrm{APF}=\mathrm{After}$ puparium formation

\section{Figure 2. Spatio-temporal expressions of Btl/FGFR and Abd-B during pupal MAG} morphogenesis

(A) Cell outlines of MAG epithelium (Fas III, grey) and cell nuclei (Topro, cyan) from a 2 day old adult after eclosion (AE), secondary cells are marked by Abd-B (Abd-B>GFP, red psuedocolor). (B$\mathrm{H})$ Expression pattern of $\mathrm{Btl}(\mathrm{btl}-\mathrm{Gal}>>U A S-G F P)$ and Abd-B (red) in the MAG-SV primordium derivates, MAG and SV, are shown from 30-90h APF. Distal tips in each image are shown at higher magnification in the bottom panel. Bright threads in D-H are the trachea. (I) Control (ci-Gal4) male reproductive system compared to (J) knockdown of $b t l(c i>b t l-R N A i)$ during MAG development marked by Actin (grey) and Topro (cyan). (K-L) shows a sample truncated MAG upon Btl knockdown, which displays loss of (L) Abd-B marked secondary cells in it. (M-N) Cartoon renditions of $(\mathrm{M})$ control and $(\mathrm{N}) \mathrm{Btl}$ knockdown are shown on the extreme right. Scale bar: $100 \mu \mathrm{m}$ Abbreviations and marking: testis (T), male accessory gland (MAG, blue broken line), seminal vesicle (SV, white dotted line), ejaculatory duct (ED, orange dotted line), ejaculatory bulb (EB, yellow dotted line).

\section{Figure 3. Hedgehog-Dpp signaling axis during MAG morphogenesis}

(A-L) Expression of $c i$ (A,D,G,J, purple), ptc (B,E,H,K, cyan blue ) and $d p p$ (C,F,I,L, orange) visualized by expressions of respective Gal4 driven UAS-GFP transgenes during budding (20h APF, boxed areas zoomed below), bud growth (30h APF) and cell differentiation (50 and 90h APF) stages. Diagram below summarizes the model of MAG developmental ground plan based on its toolkit expressions. Bt1/FGFR is hallmark of the tube formation and Abd-B of tube specialization stage, 
Developmental ground plan of Drosophila male accessory gland

383 while Hh-Dpp signaling toolkit is common to both stages. Scale bar: $100 \mu \mathrm{m}$ Marking scheme of the

384 individual organs are as in preceding Figure 2.

385

386 Figure 4: Common developmental ground plan of Drosophila MAG and mammalian prostate

387 (A-C) Cartoon representation of MAG development from Bt1/FGFR primordial cells: (A) unitary

388 MAG and SV buds with a priori lumen appear from the MAG-SV primordium. (B) MAG grows as a

389 short tube. (C) MAG eventually connects caudally with the ejaculatory duct. (D) Illustration of MAG

390 cross-section with two differentiated cell types, secondary cells are marked by Abd-B (E-G) Cartoon

391 representation of mouse prostate development: (E) prostatic buds and SV are separate (F) individual

392 primary prostatic bud does not have a lumen $(\mathrm{G})$ branching with each individual tips marked by

393 FGFR, while canalization precedes to form their lumen $(\mathrm{H})$ Illustration of prostatic cross-section

394 depicting two differentiated cell types, luminal cells are marked by Hoxb13. 
Figure 1

110-120h AEL
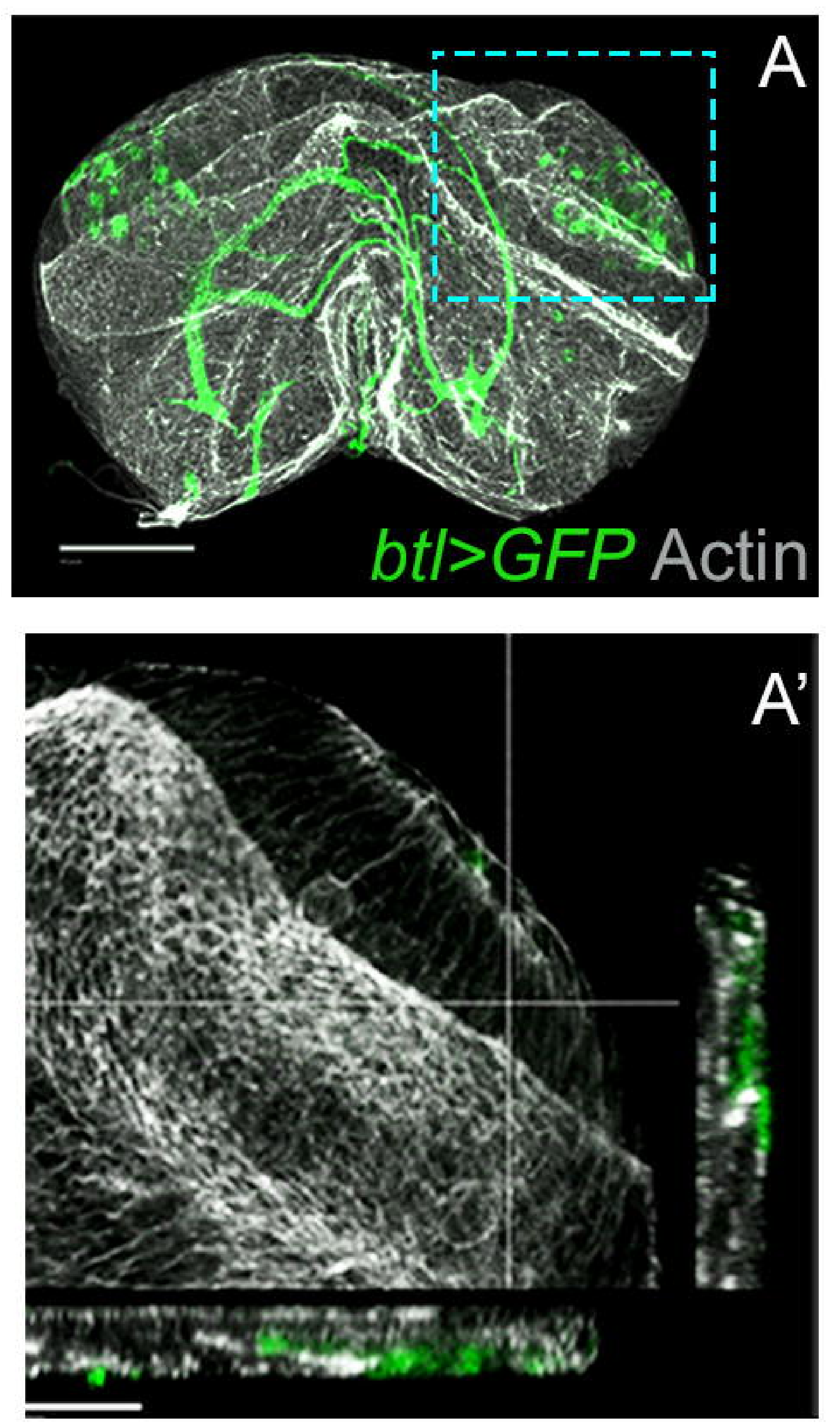

Oh APF
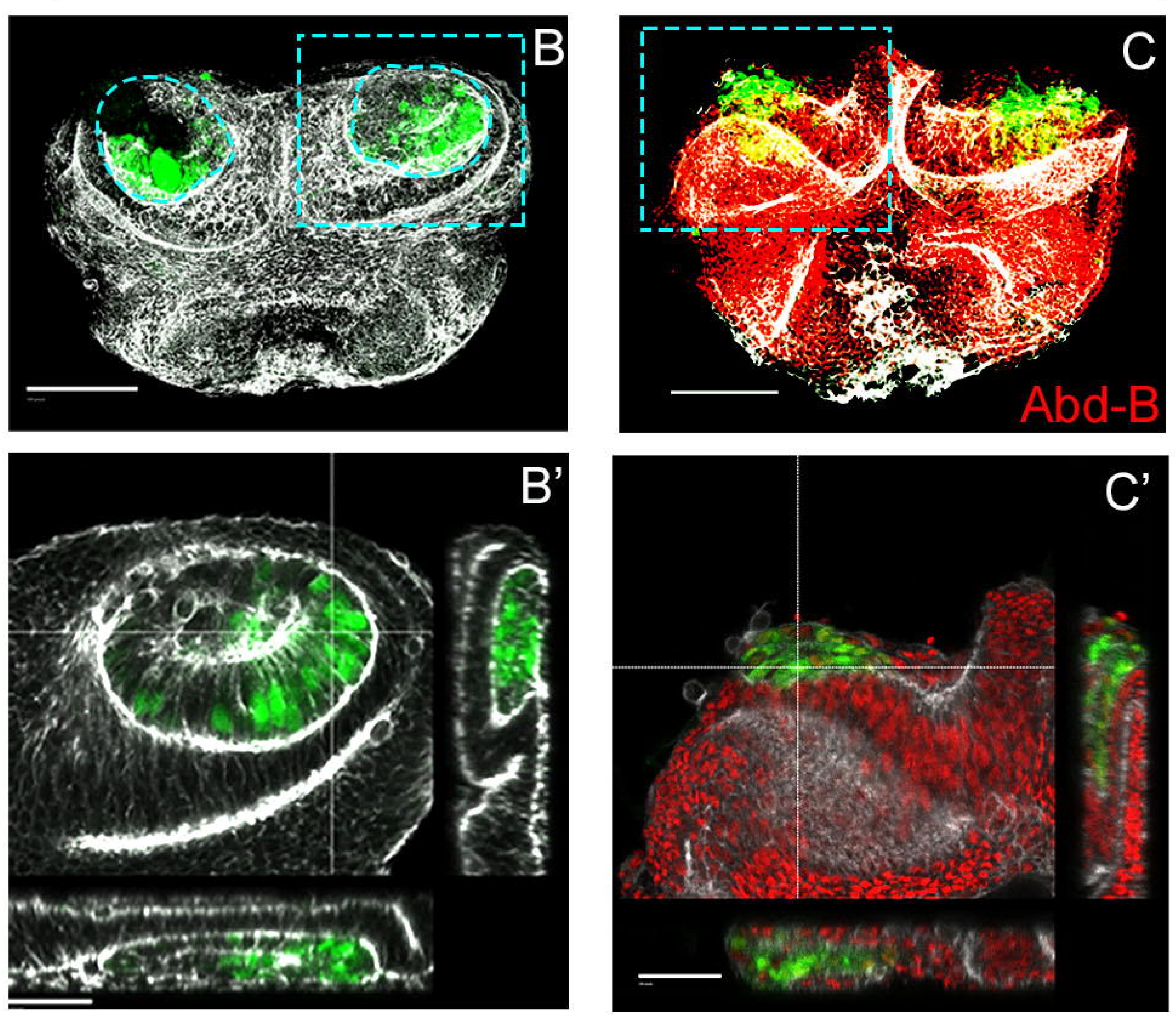
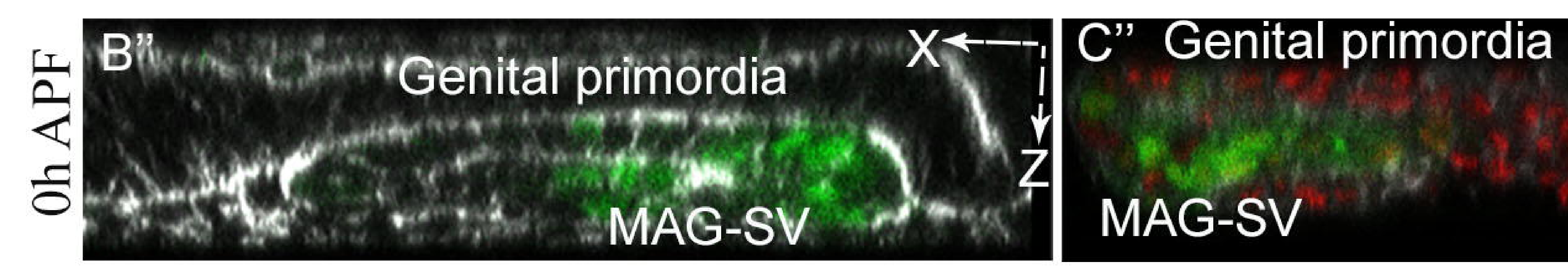

Oh APF
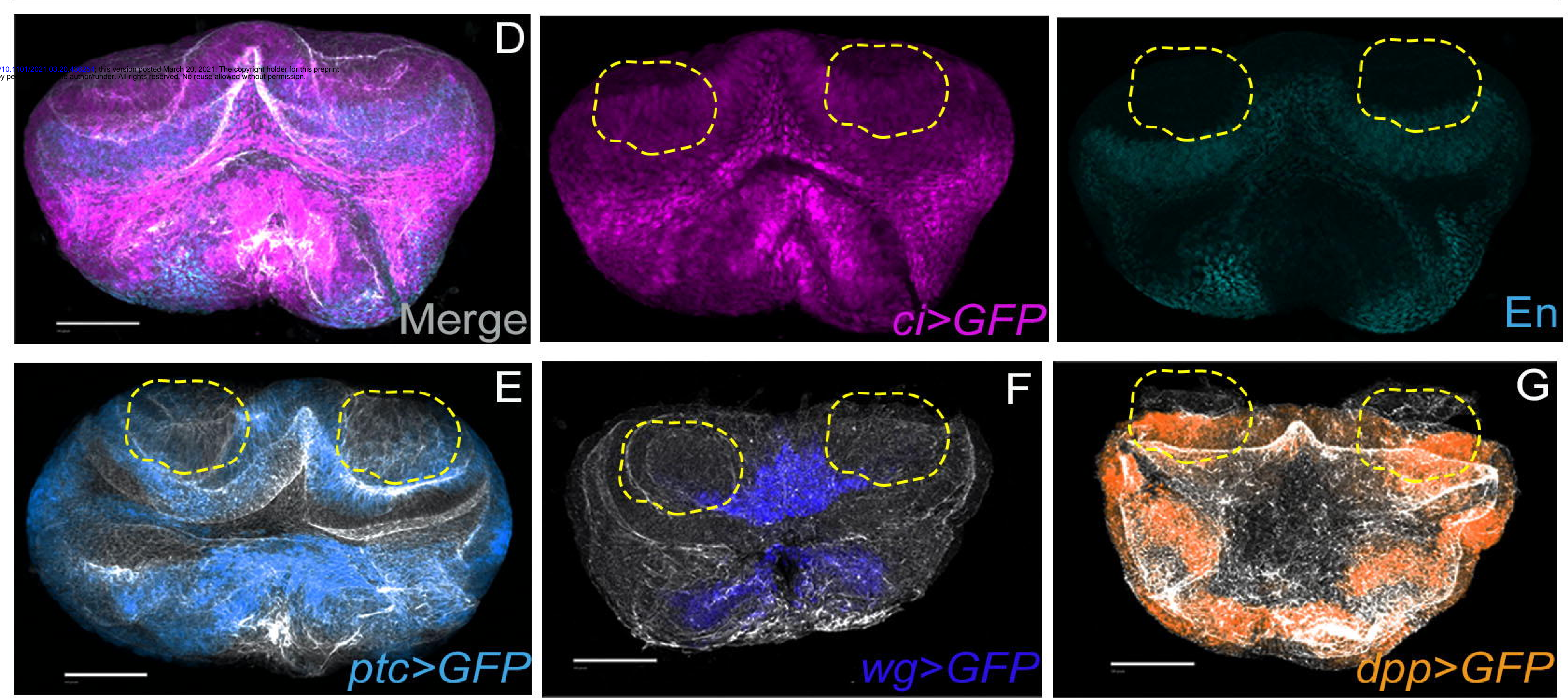
Figure 2
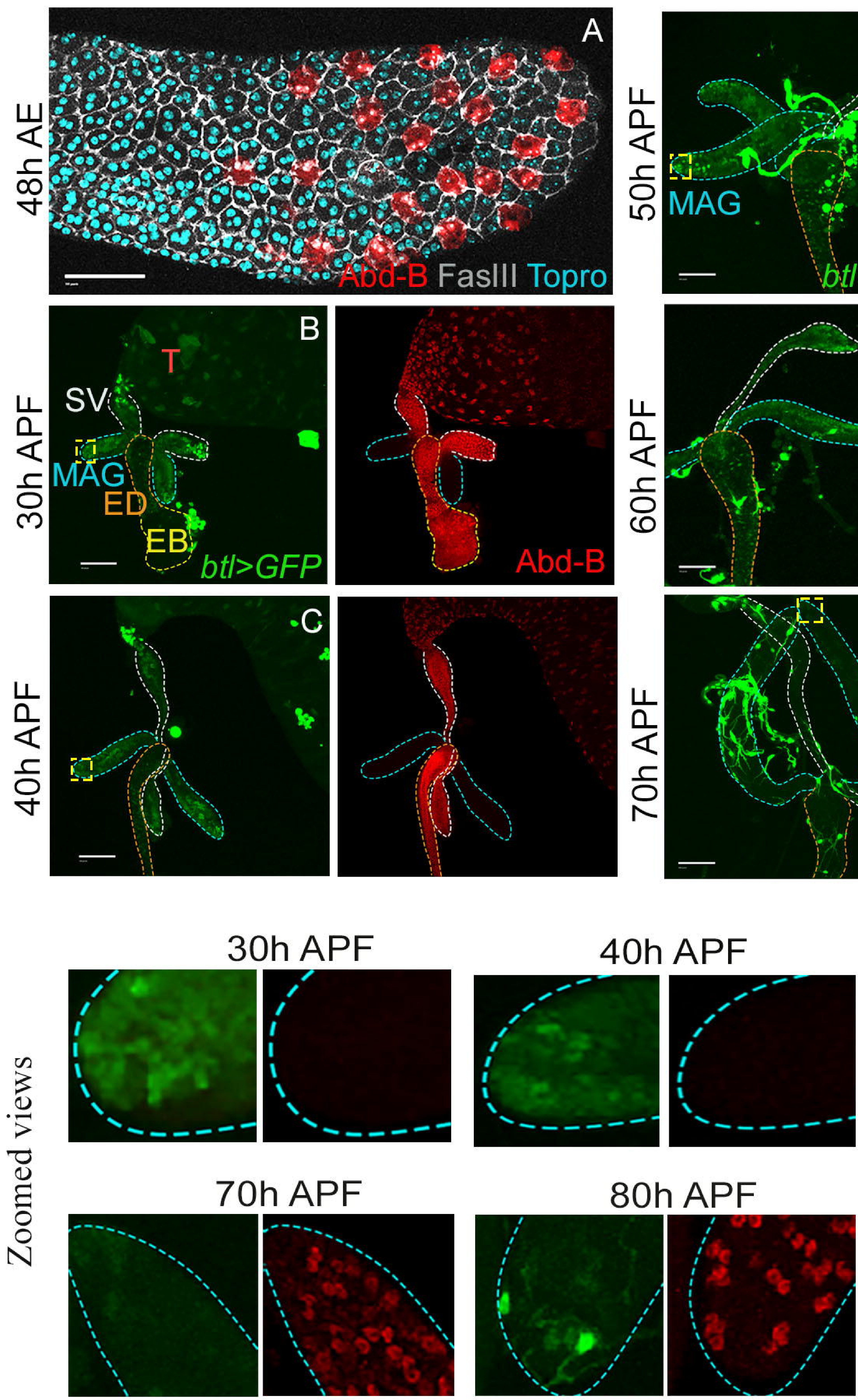

ci>+

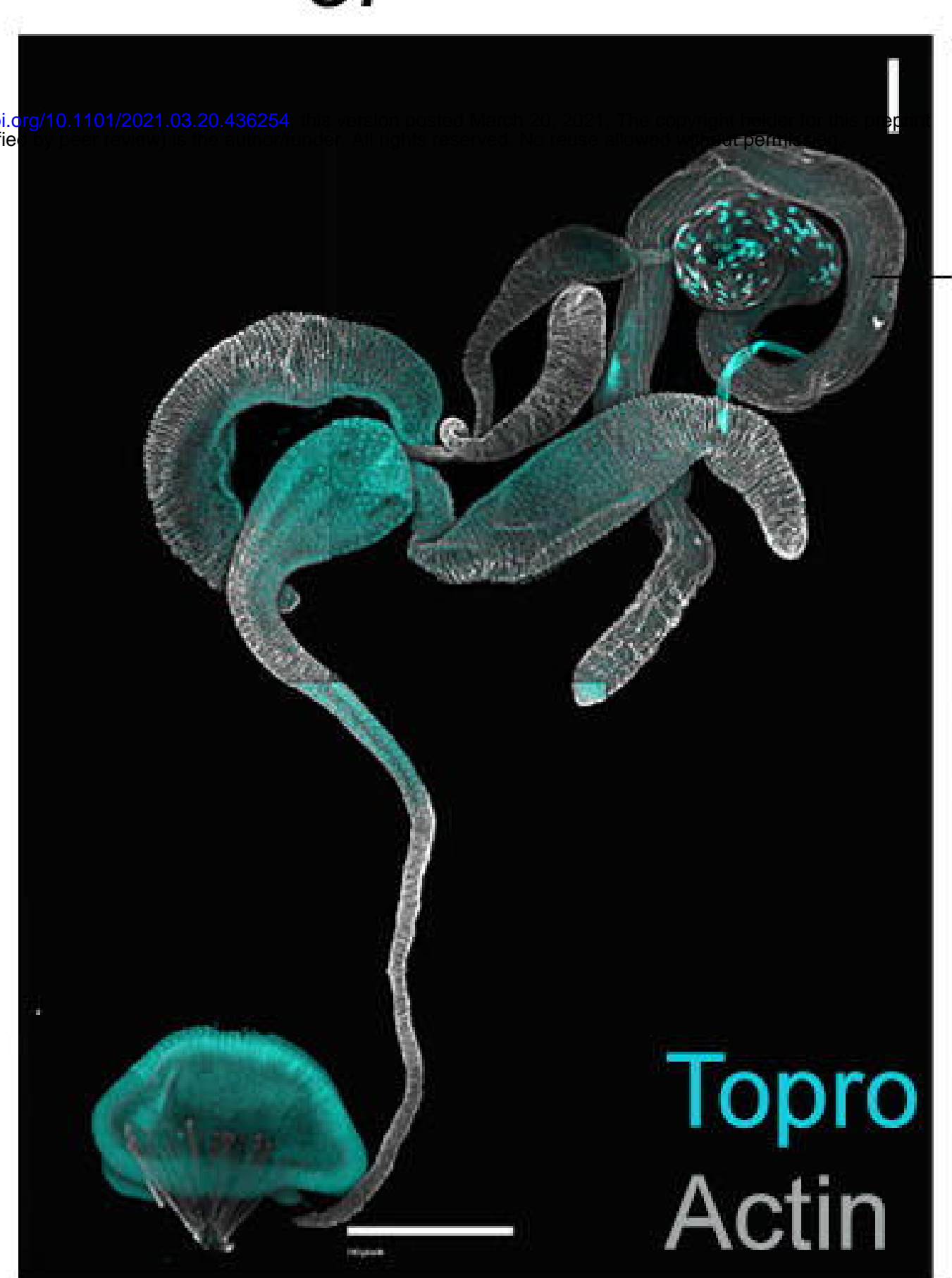

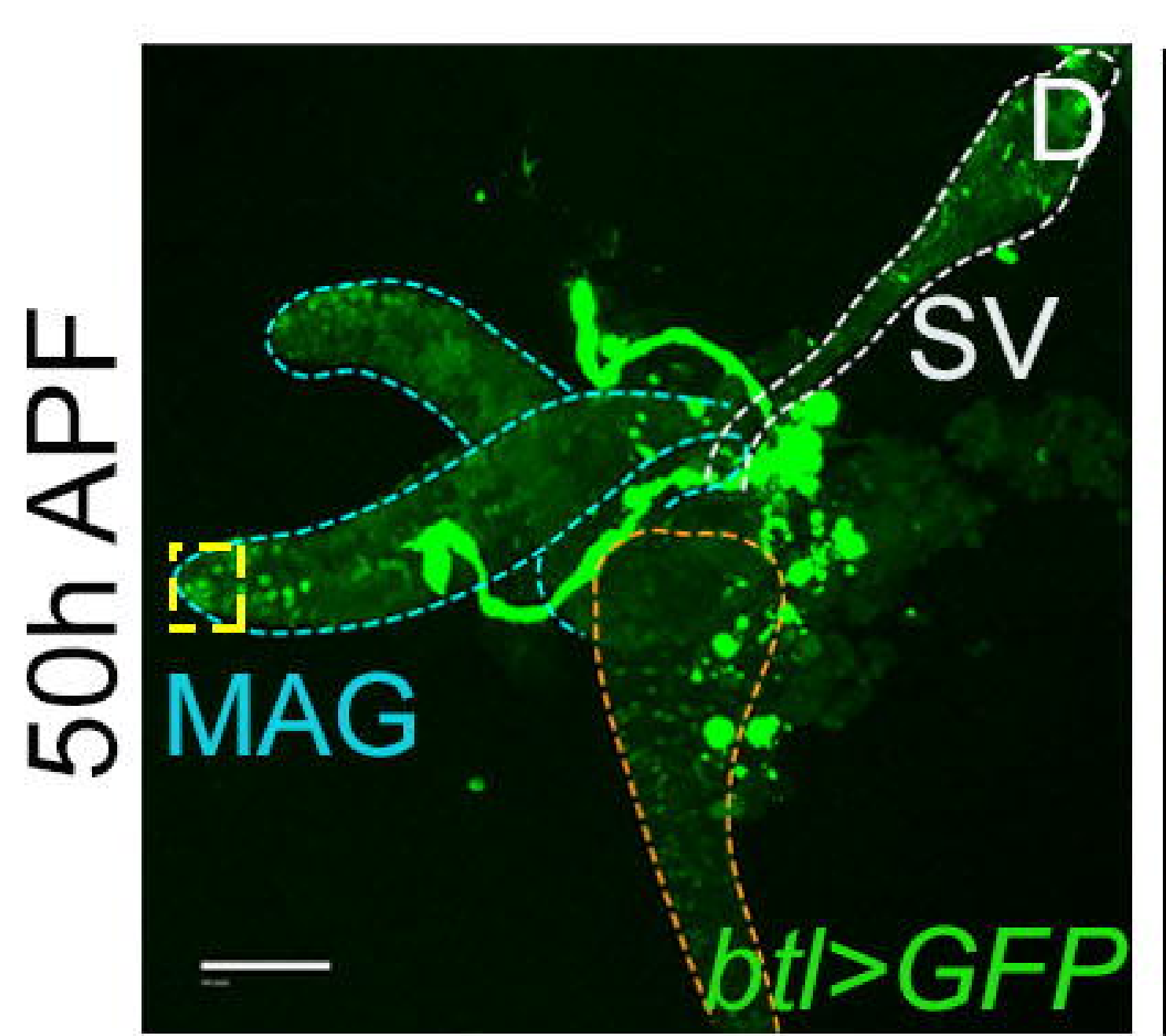
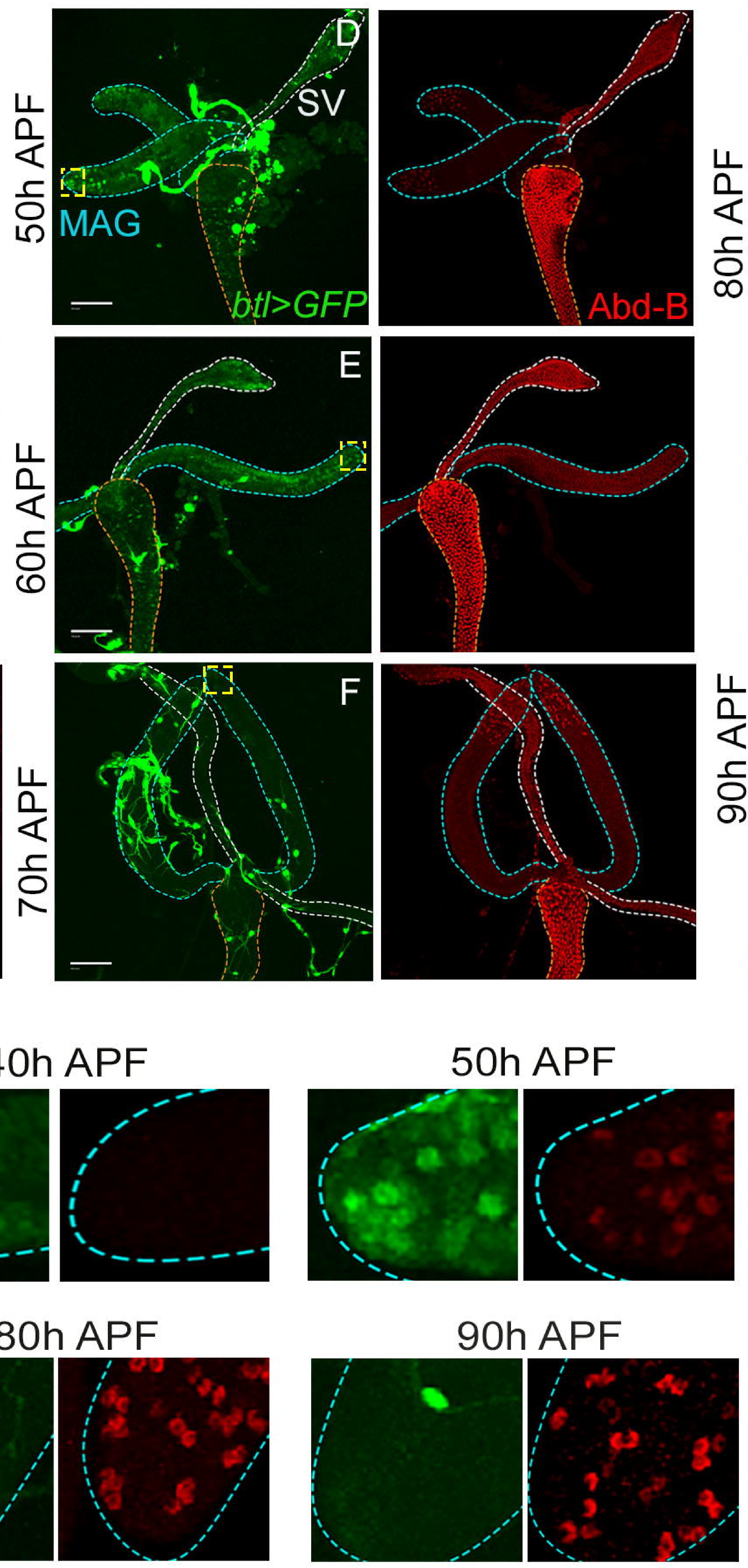

M

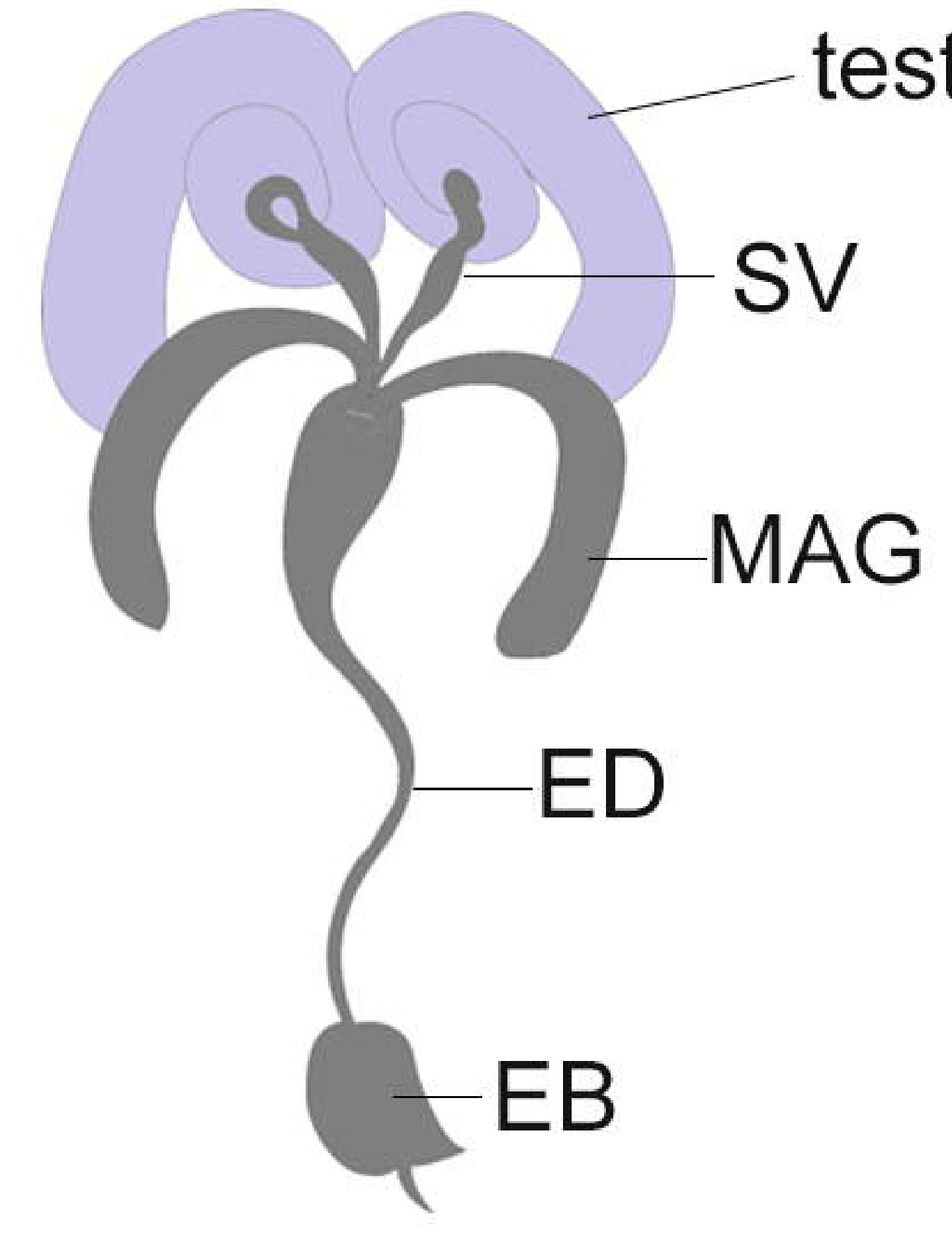

Wild-type
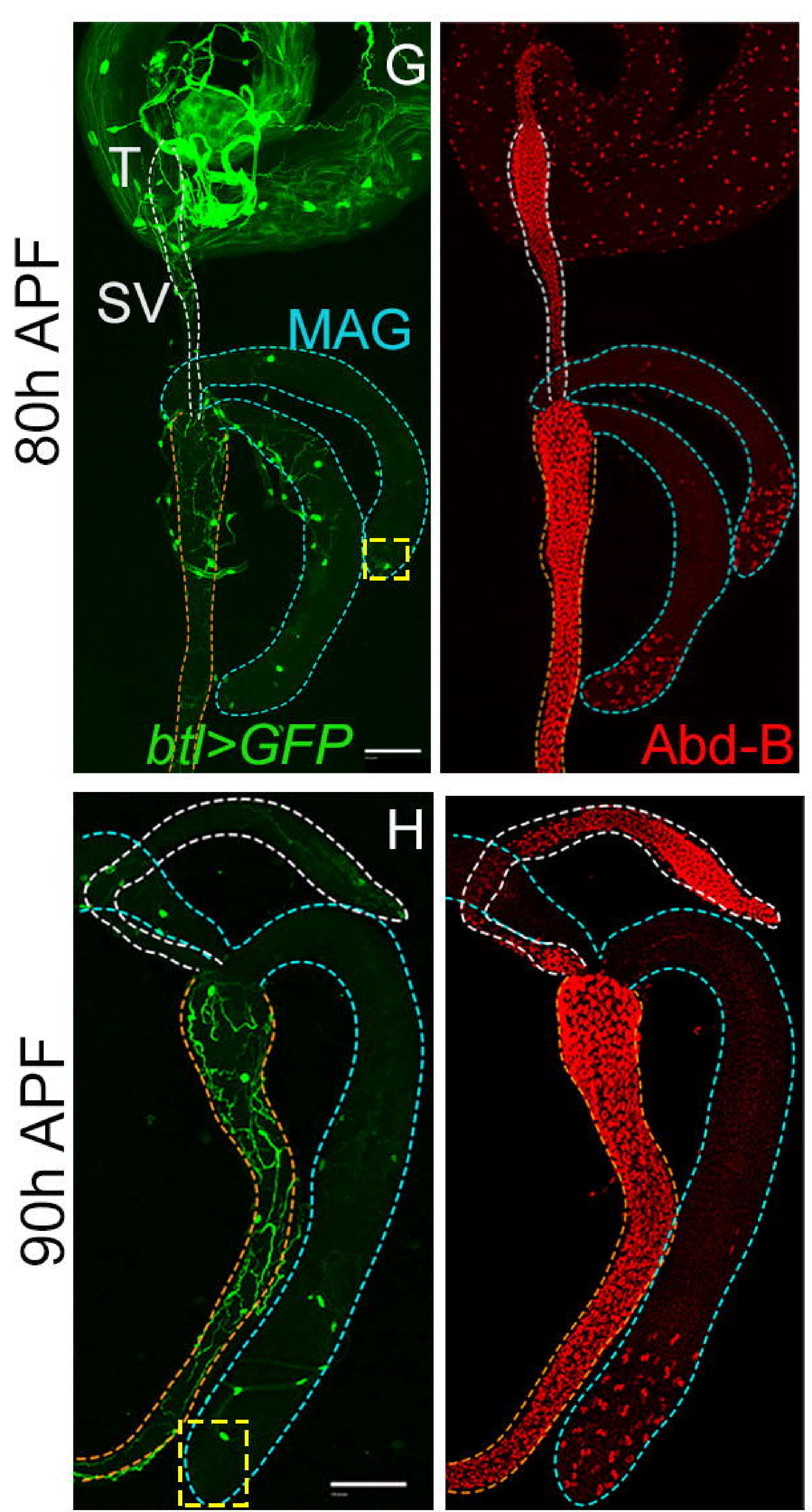

60h APF
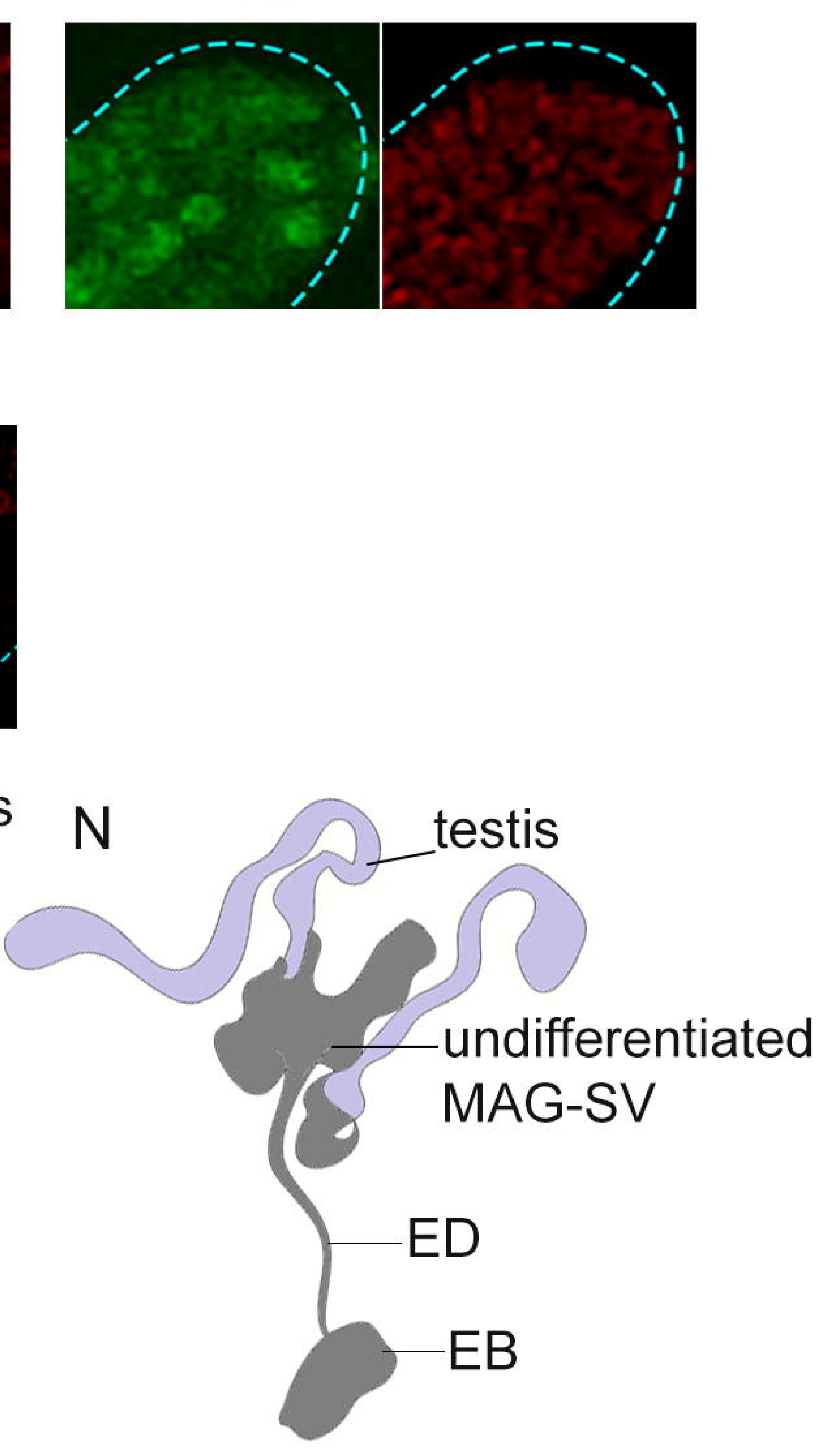

BtI/FGFR knockdown 
Figure 3
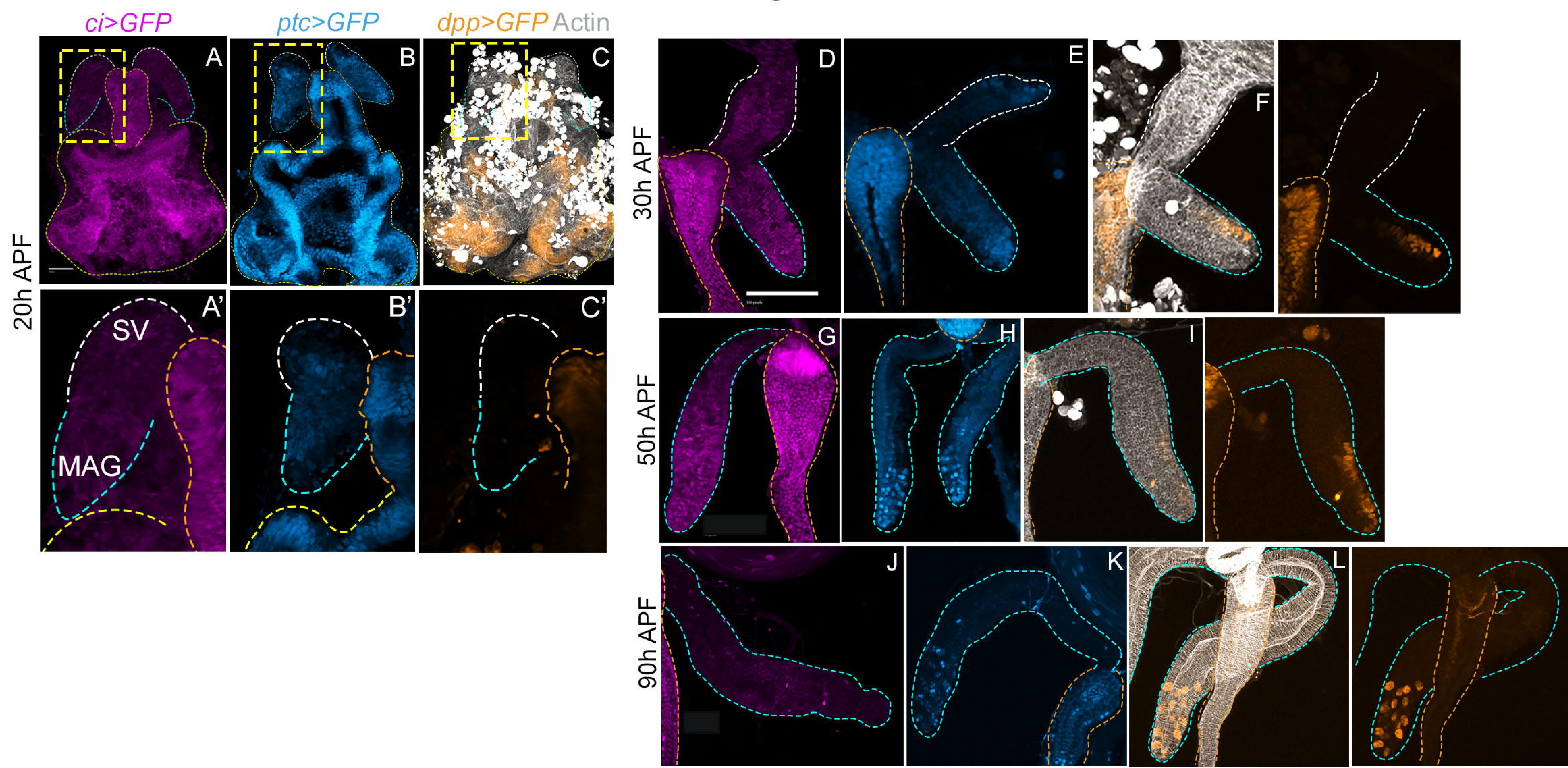

Tube formation

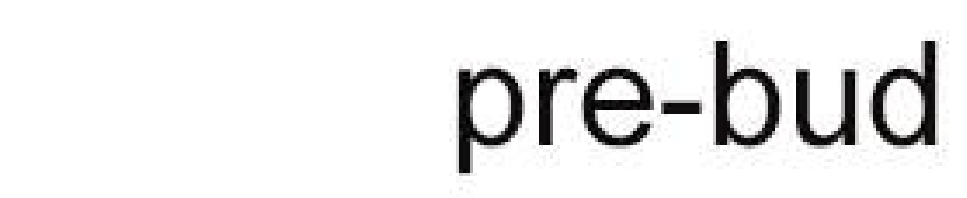$$
\text { bud growth }
$$

$$
\text { budding }
$$

Tube specialization

cell differentiation

$50 \mathrm{~h}$

$60 \mathrm{~h}$
$70 \mathrm{~h}$

$80 \mathrm{~h}$

$90 \mathrm{~h}$

Abd-B

$\mathrm{Ci}$

En

Ptc

$\mathrm{Wg}$

Dpp 
Figure 4

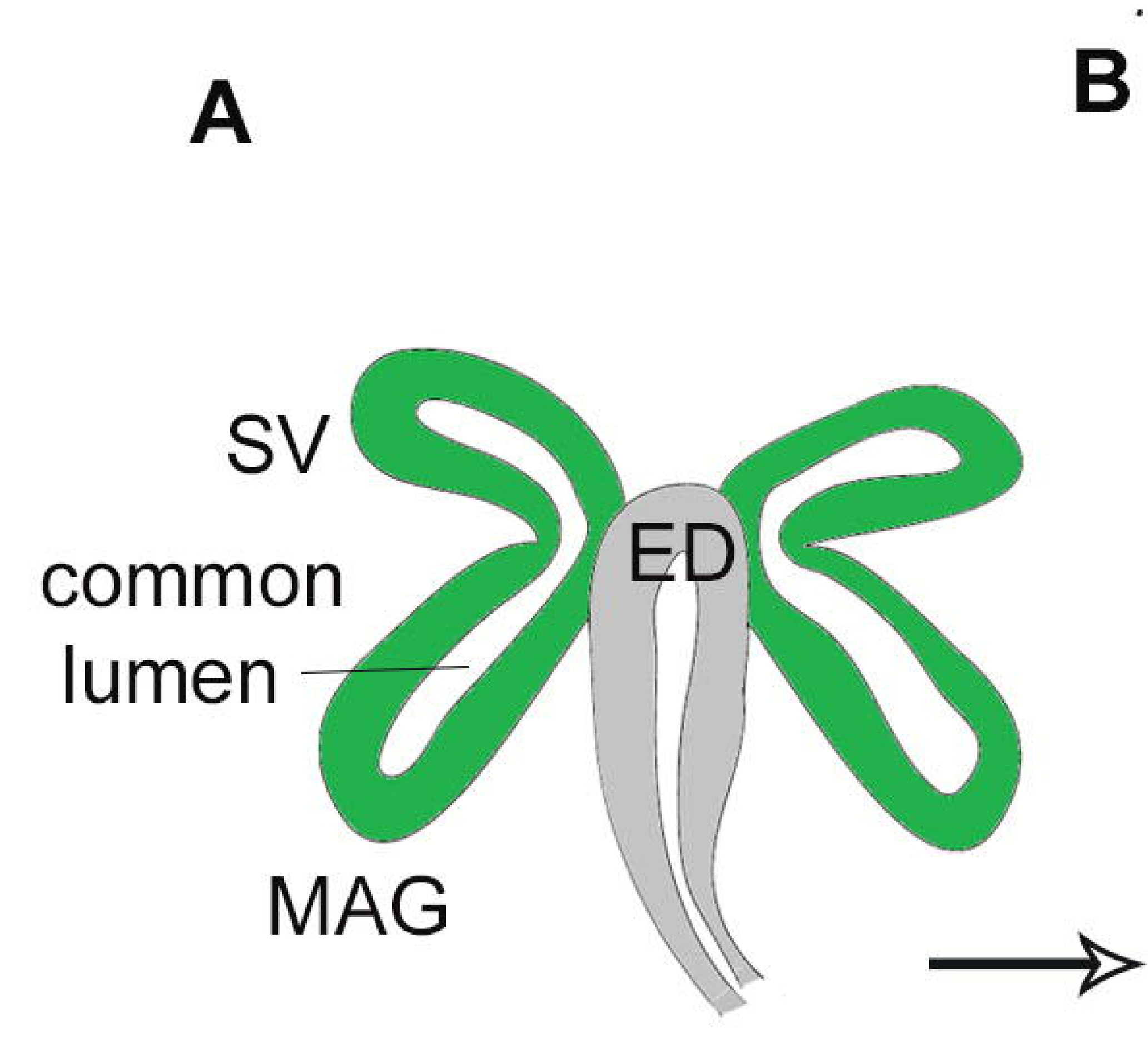

Btl/FGFR

B

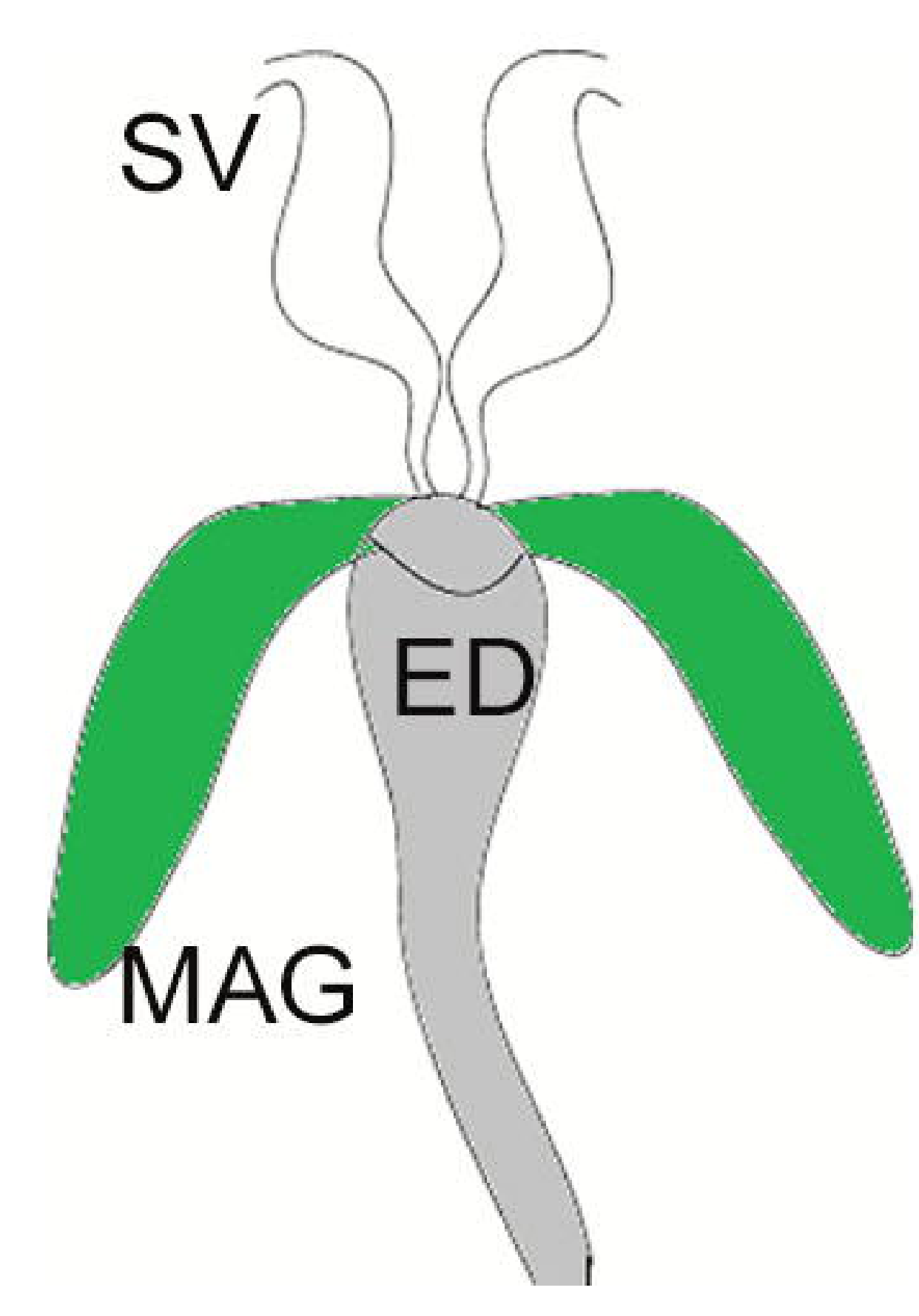

C

D

\section{Drosophila MAG}

E

F

G

H

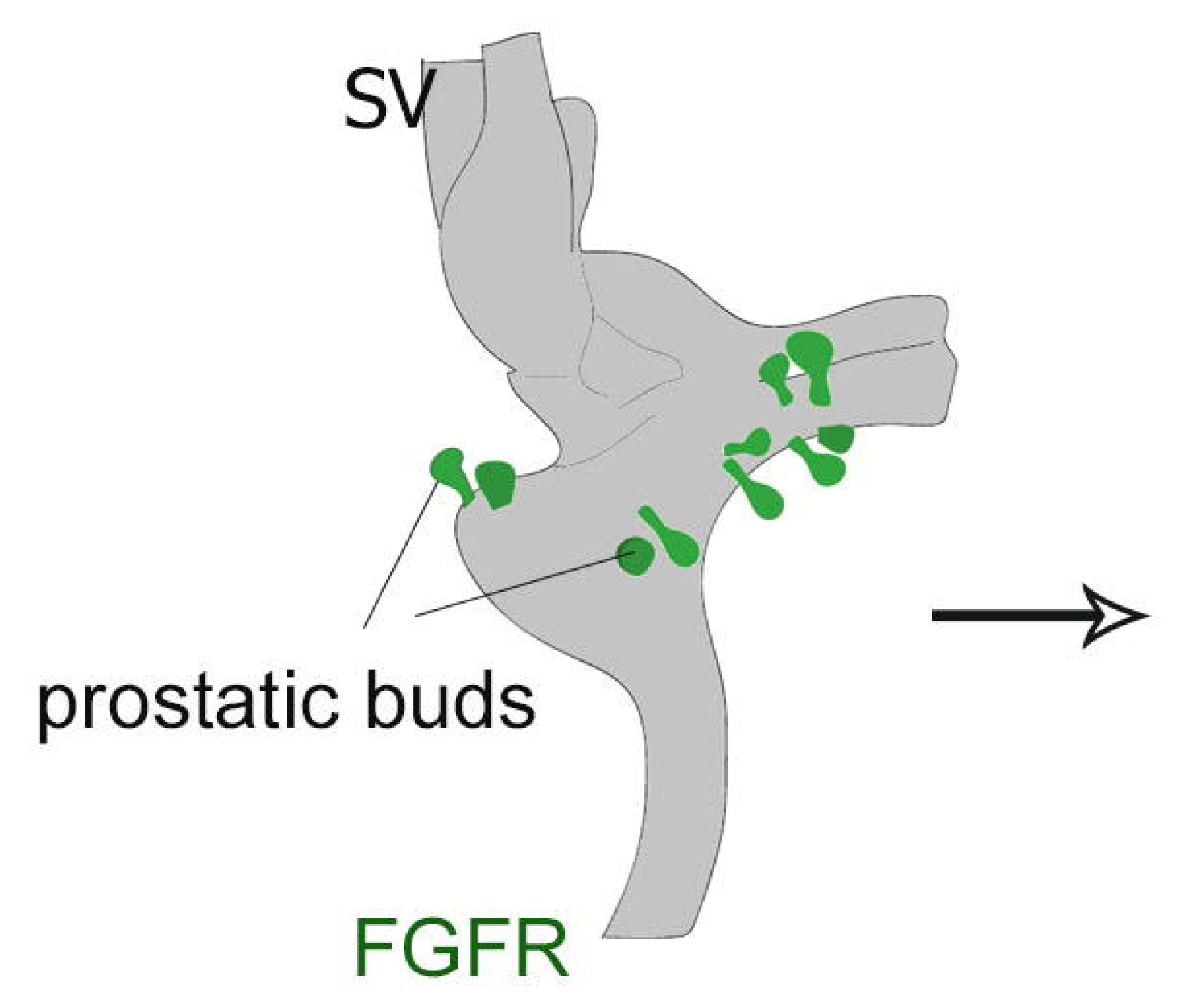
branching tips

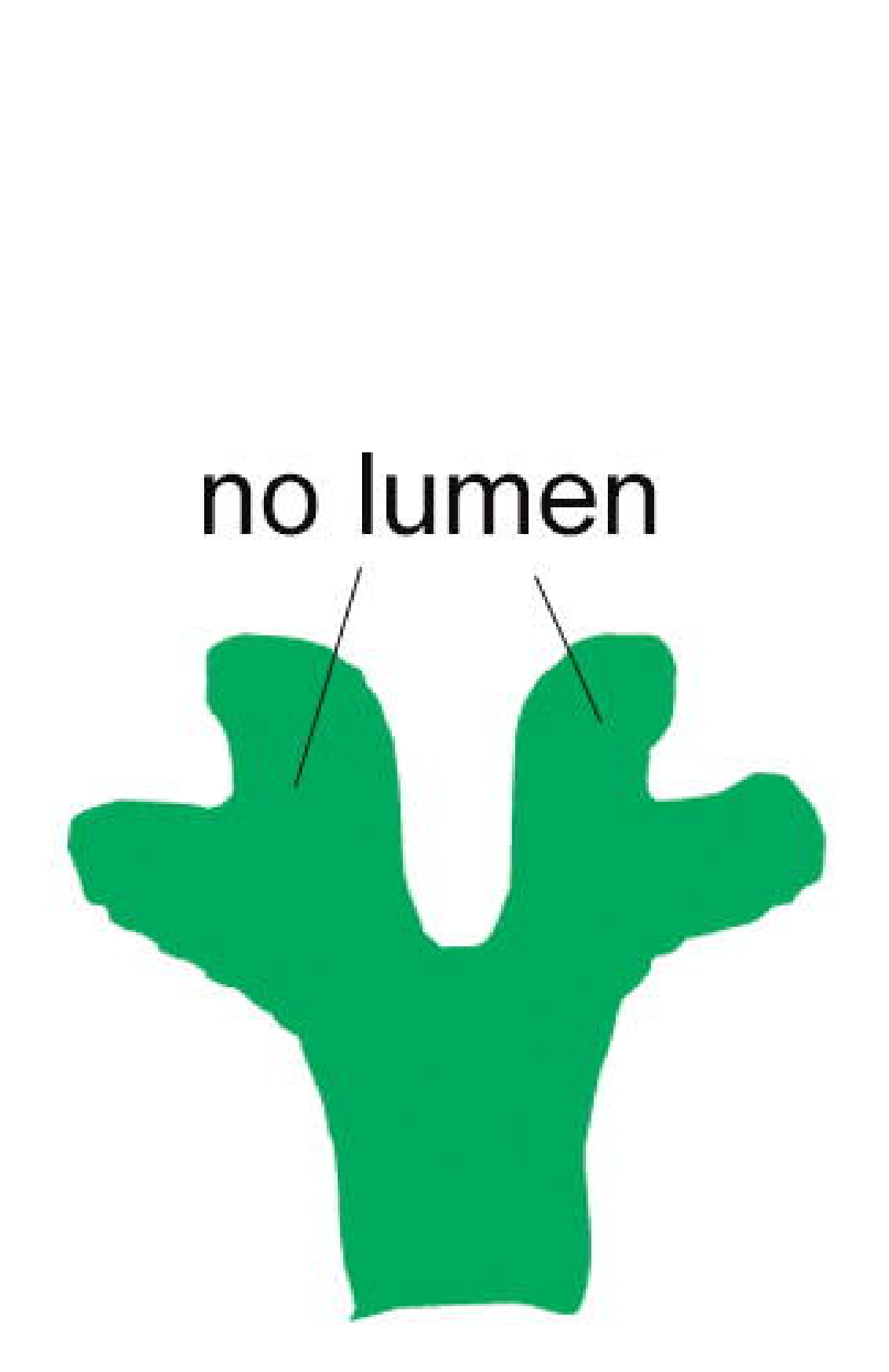

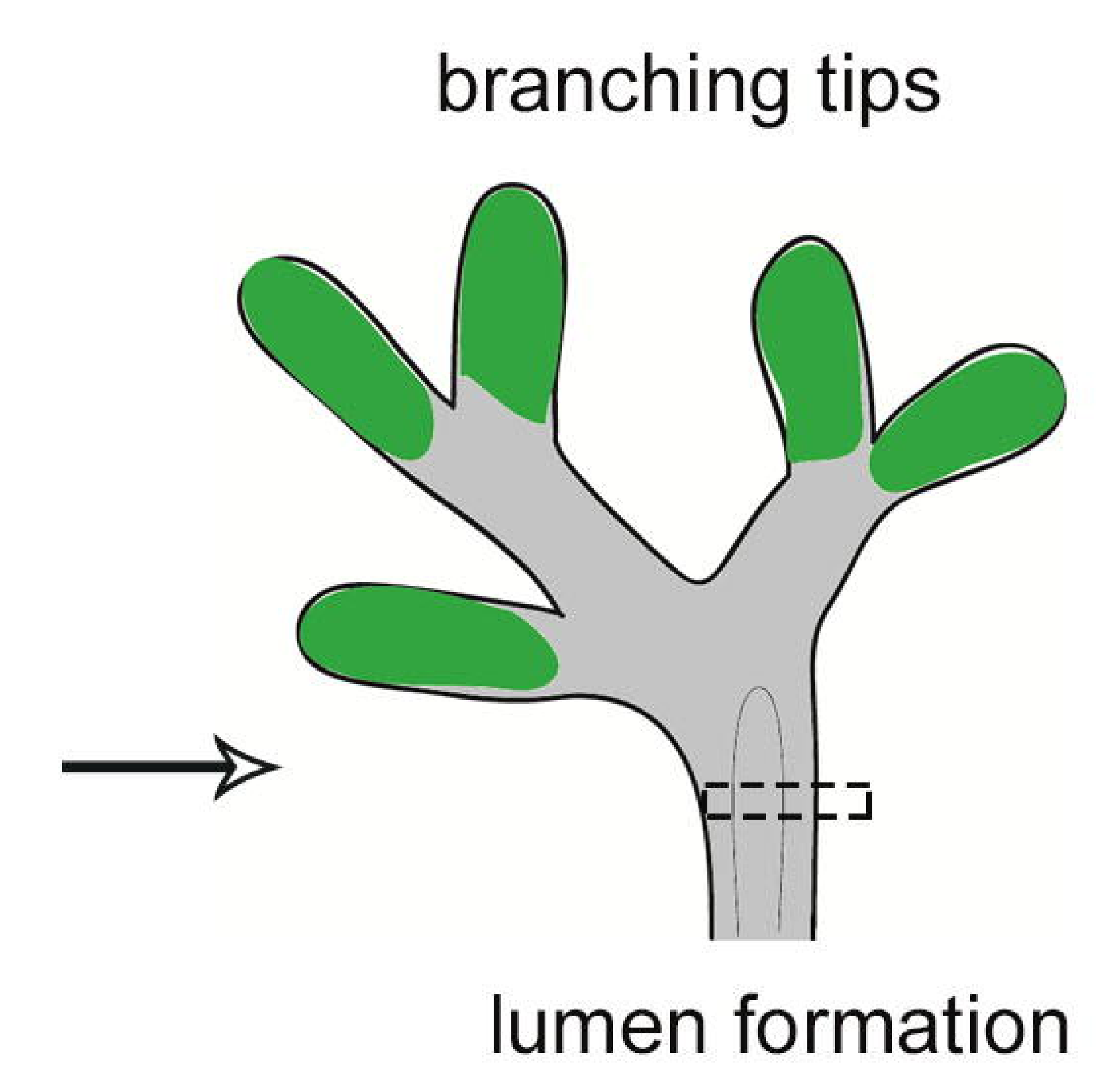

Mouse prostate 